Physics International, 2012, 3 (2), 50-57

ISSN: 1948-9803

(C)2012 Science Publication

doi:10.3844/pisp.2012.50.57 Published Online 3 (2) 2012 (http://www.thescipub.com/pi.toc)

\title{
The Gravity Field of a Cube
}

\author{
${ }^{1}$ James Michael Chappell, ${ }^{2}$ Mark John Chappell, ${ }^{1}$ Azhar Iqbal and ${ }^{1}$ Derek Abbott \\ ${ }^{1}$ School of Electrical and Electronic Engineering, \\ Faculty of Engineering, Computer and Mathematical Sciences, \\ University of Adelaide, Adelaide, SA 5005, Australia \\ ${ }^{2}$ Applied Cognitive Neuroscience Research Unit, \\ Griffith Health Institute, Griffith University, Queensland, 4111, Australia
}

\begin{abstract}
We calculate the Newtonian gravitational potential and field of a cubic, homogeneous asteroid and we apply it to the orbit of possible satellites. Large astronomical objects such as stars or planets, naturally tend to form spherical shapes due to the dominance of the gravitational forces, but as a thought experiment, we consider the properties of a planet in the form of a perfect cube. We investigate the formation of stable orbits around such cubic objects, for the case of a static as well as a rotating cube employing the method of Poincare sections. The calculation of the gravitational field around non-spherical objects has a significant role in space missions to investigate asteroid belt objects that require calculating orbits around a large non-spherical mass. The calculation of such non-spherical fields also has relevance in identifying deposits or beds of ores inside the Earth, by measuring gravitational anomalies.
\end{abstract}

Keywords: Gravitational Field, Cube, Satellite Orbits, Poincare Sections

\section{INTRODUCTION}

Much work has now been carried out in calculating the gravitational field around cuboid objects and so we investigate in this study, the nature of the gravitational field and satellite orbits around a hypothetical cubic Earth-size planet. Plato, in the Timaeus, links what he considers the four basic elements, fire, air, water and earth, with the four regular solids, using the tetrahedron to represent fire, the octahedron to represent air, the icosahedron to represent water and the cube to represent earth. Taking Plato's idea literally, we might imagine the Earth in the form of a perfect cube. We can then calculate the gravitational field around this object, the process being simplified by the simple endpoints needed when integrating over a right rectangular prism, assuming Newton's classical law of gravitation. The equipotentials of the field will give us, for example, the shape of lakes that would form on the face of such a cube. The concept of cubical planets is in fact not new, as in 1884 the Swiss astronomer Arndt claimed to have discovered a cubical planet orbiting beyond the orbit of Neptune, though unfortunately the discovery was never verified (Vankirk,
1885). The appearance of cubic planets in our universe may appear extremely improbable, however if gravity acted over the $L^{\infty}=\max _{\mathrm{i}=1 \ldots 3}\left|\mathrm{x}_{\mathrm{i}}\right|$ metric rather than the usual Euclidean metric then cubic planets would naturally form under the force of gravity.

The gravitational field of a cube was apparently first calculated in (MacMillan, 1958) and later extended to cuboid masses (Nagy, 1966; Mufti, 2006a), including varying density (Garcia-Abdeslem, 2005; Hansen, 1999) and also for general polyhedra (Paul, 1974; Coggon, 1976), as well as for a range of simpler planar objects such as straight line segments as well as disks and annular shapes (Riaguas et al., 1999; 2001; Azevedo and Ontaneda, 2007; Azevedo et al., 2005; Fukushima, 2010; Alberti and Vidal, 2007; Blesa, 2005; Gutierrez-Romero et al., 2004; Palacian et al., 2006; Najid and Elourabi, 2012; Najid et al., 2012; Iorio, 2007; 2012). These results were found to have application in the calculation of the gravitational anomalies on the Earth (Mufti, 2006b) and the slowdown of the Earth's rotation rate due to tidal drag (Celnikier, 1990). Further investigations explored the nature of the satellite orbits that would form around a

Corresponding Author: James Michael Chappell, School of Electrical and Electronic Engineering, Faculty of Engineering, Computer and Mathematical Sciences, University of Adelaide, Adelaide, SA 5005, Australia 
static cube (Liu et al., 2011a; Werner, 1994), as well as for rotating cubes (Liu et al., 2011b) using simulations and the method of Poincare sections (Liu et al., 2011a; Scheeres et al., 2000). These preliminary investigations of orbits around cubic objects are of significance as they can be considered the first step towards an analysis of orbits around more general shaped bodies. These investigations, for example, have application to future missions to investigate asteroids, where orbits need to be calculated around rotating non-spherical objects (Liu et al., 2011a; Werner and Scheeres, 1996; Michalodimitrakis and Bozis, 1985; Liu et al., 2011b; Scheeres et al., 1998; 1996; Yu and Baoyin, 2012a; 2012b; 2012c).

In this study we firstly produce a calculation for the field of a perfect cube or rectangular solid of uniform density, with which we then investigate the formation of stable orbits around such objects using the method of Poincare sections, before concluding with some lighthearted remarks regarding the nature of life on a hypothetical cubic planet.

\subsection{Analysis}

\subsubsection{Gravitational Potential of a Cube}

We first seek the Newtonian gravitational potential of a rectangular solid of uniform density $\rho$ with Newton's universal gravitational constant $G$. We suppose the rectangular solid has a length $2 \mathrm{~L}$, breadth $2 \mathrm{~B}$ and depth $2 \mathrm{D}$, oriented along the $\mathrm{x}, \mathrm{y}, \mathrm{z}$ axes respectively, centered on the origin, then we have the potential Equation 1:

$$
\begin{aligned}
& \mathrm{V}(\mathrm{X}, \mathrm{Y}, \mathrm{Z})= \\
& -\mathrm{G} \rho \int_{-\mathrm{D}}^{\mathrm{D}} \int_{-\mathrm{B}}^{\mathrm{B}} \int_{-\mathrm{L}}^{\mathrm{L}} \frac{\mathrm{dx^{ \prime } d y ^ { \prime } \mathrm { dz }}}{\sqrt{\left(\mathrm{X}-\mathrm{x}^{\prime}\right)^{2}+\left(\mathrm{Y}-\mathrm{y}^{\prime}\right)^{2}+\left(\mathrm{Z}-\mathrm{z}^{\prime}\right)^{2}}} \\
& =-G \rho \int_{Z=-D-Z}^{D-Z} \int_{y=-B-Y}^{B-Y} \int_{x=-L-X}^{L-X} \frac{d x d y d z}{\sqrt{x^{2}+y^{2}+x^{2}}} \\
& =-\mathrm{G} \rho \int_{\mathrm{x}=-\mathrm{L}-\mathrm{X}}^{\mathrm{L}-\mathrm{X}} \int_{\mathrm{y}=-\mathrm{B}-\mathrm{Y}}^{\mathrm{B}-\mathrm{Y}}\left[\operatorname{In}\left(\mathrm{z}+\sqrt{\mathrm{x}^{2}+\mathrm{y}^{2}+\mathrm{z}^{2}}\right)\right]_{\mathrm{z}=-\mathrm{D}-\mathrm{Z}}^{\mathrm{D}-\mathrm{Z}} \mathrm{dydx},
\end{aligned}
$$

where, we made the substitution, $x=x^{\prime}-X, y=y^{\prime}-Y$ and $z$ $=z^{\prime}-Z$ and completed the integral over the $\mathrm{z}$ coordinate. Next, integrating over the $y$ variable and using $r=\sqrt{x^{2}+y^{2}+z^{2}}$, we find Equation 2:

$$
\begin{aligned}
& \mathrm{V}=-\mathrm{G} \rho \int_{\mathrm{x}=-\mathrm{L}-\mathrm{X}}^{\mathrm{L}-\mathrm{x}} \mathrm{dx}[[\mathrm{y} \ln (\mathrm{z}+\mathrm{r})+\mathrm{z} \ln (\mathrm{y}+\mathrm{r}) \\
& \left.\left.-\mathrm{y}+\mathrm{x} \arctan \frac{\mathrm{y}}{\mathrm{x}}-\mathrm{x} \arctan \frac{\mathrm{yz}}{\mathrm{xr}}\right]_{\mathrm{z}=-\mathrm{D}-\mathrm{Z}}^{\mathrm{D}-\mathrm{Z}}\right]_{\mathrm{y}=-\mathrm{B}-\mathrm{Y}}^{\mathrm{B}-\mathrm{Y}}
\end{aligned}
$$

and with the integral over $\mathrm{x}$, we achieve our final result:

$\mathrm{V}(\mathrm{X}, \mathrm{Y}, \mathrm{Z})=-G \rho$

$\left[\left[\left[\begin{array}{l}y z \ln (x+r)-\frac{x^{2}}{2} \arctan \frac{y z}{x r}+x z \ln \\ (y+r)-\frac{y^{2}}{2} \arctan \frac{x z}{y r}+x y \ln (z+r) \\ -\frac{z^{2}}{2} \arctan \frac{x y}{z r}\end{array}\right]_{z=-D-Z}^{D-Z}\right]_{y=-B-Y}^{\text {R-Y }}\right]_{X=-L-X}^{L-X}$

using $\mathrm{x}_{1}=\mathrm{x}, \mathrm{x}_{2}=\mathrm{y}, \mathrm{x}_{3}=\mathrm{z}$ and $\mathrm{D}_{1}=\mathrm{L}, \mathrm{D}_{2}=\mathrm{B}, \mathrm{D}_{3}=\mathrm{D}$ we can write Equation 4:

$V\left(X_{1}, Y_{2}, Z_{3}\right)=-G \rho \sum_{j=1}^{3}\left[\sum_{i=1}^{3}\left(\begin{array}{l}\frac{v}{x_{i}} \ln \left(x_{i}+1\right) \\ \left.-\frac{x_{i}^{2}}{2} \arctan \frac{v}{x_{i}^{2} r}\right)\end{array}\right]\right]_{x_{j}=-D_{j}-x_{j}}^{D_{j}-x_{j}}$

For the gravitational potential of a right rectangular prism mass, where $\mathrm{v}=\mathrm{x}_{1} \mathrm{x}_{2} \mathrm{x}_{3}$. The pairs of $\log$ and arctan terms combine to produce the expected $\frac{1}{\mathrm{r}}$ falloff in gravitational potential at large distances. For example, for a $2 \times 2 \times 2 \mathrm{~m}^{3}$ cube, with $\mathrm{G} \rho=1$, as $\mathrm{x} \rightarrow \infty$, $\mathrm{yz} \ln (\mathrm{x}+\mathrm{r})$ $\frac{\mathrm{x}^{2}}{2} \arctan \frac{\mathrm{yz}}{\mathrm{xr}} \rightarrow-\frac{8}{\mathrm{r}}$, as expected for a point source.

In order to reveal the equipotentials around this object, we can imagine a very low density fluid being added to a face of a cube and by plotting Equation (3) at a constant potential, we find a lake as shown in Fig. 1. A perfect circle is shown for comparison and we can see how the water is pulled up towards the corners due to the extra mass present there.

The surface of the lake, for someone boating on the surface, will be approximately spherical in shape, as it is for a large lake upon the Earth, as is also shown in Fig. 1, found by plotting Equation (3) at a constant potential in three dimensions.

\subsection{Gravitational Field}

We calculate the gravitational field vectors from Equation 5:

$$
\vec{g}=-\nabla V=-\left(\frac{\partial V}{\partial x} e_{1}+\frac{\partial V}{\partial y} e_{2}+\frac{\partial V}{\partial z} e_{3}\right)
$$



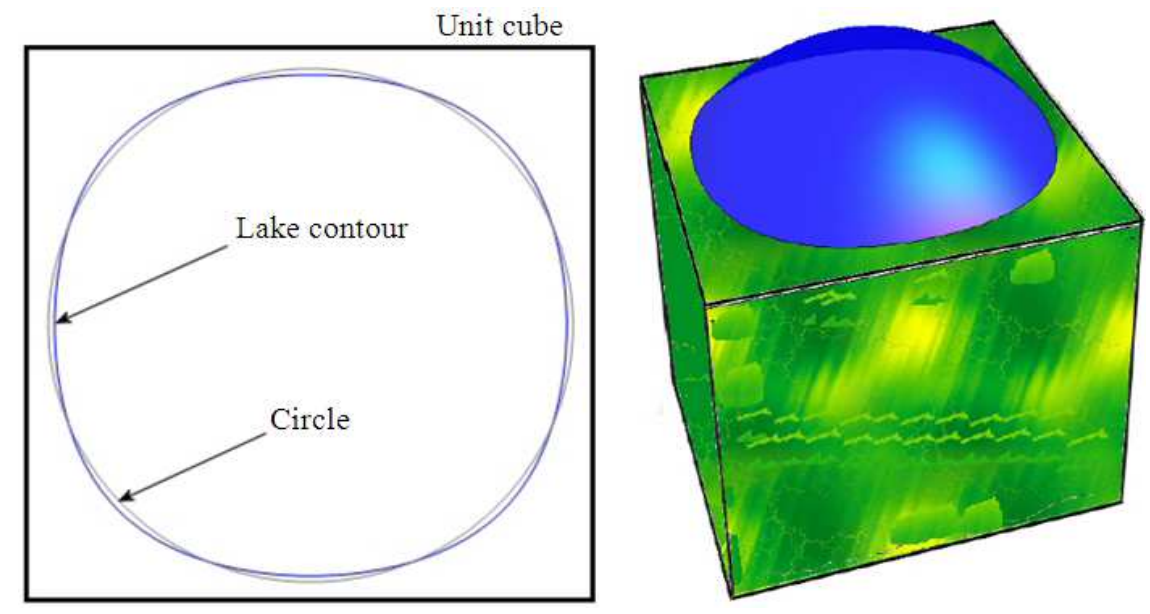

Fig. 1. A lake formed on the surface of a cube. As expected, the edge of the lake is 'pulled up' towards the corners, due to the extra mass present there, but forming a nearly spherical surface

The field vector in the $\mathrm{x}$ direction $\mathrm{g}_{\mathrm{x}}=-\frac{\partial \mathrm{v}}{\partial \mathrm{x}}$, can be deduced from Equation (2), before the last integral is calculated and hence by the fundamental theorem of calculus we find Equation 6:

$$
\left.g_{x}=G \rho \sum_{j=1}^{3} y \ln (z+r)+z \ln (y+r)-x \arctan \frac{y z}{x r}\right]_{x_{j}=-D_{j}-x_{j}}^{D_{j}-x_{j}}
$$

and from symmetry we can also easily deduce the field strengths in the $\mathrm{y}$ and $\mathrm{z}$ directions, giving the field strength vector $\vec{g}=\sum_{i=1}^{3} g_{i} e_{i}$, where Equation 7:

$$
\begin{aligned}
& \mathrm{g}_{\mathrm{i}}= \\
& \left.\mathrm{G} \rho \sum_{\mathrm{b}=1}^{3} \mathrm{x}_{\mathrm{j}} \ln \left(\mathrm{x}_{\mathrm{k}}+\mathrm{r}\right)+\mathrm{x}_{\mathrm{k}} \ln \left(\mathrm{x}_{\mathrm{j}}+\mathrm{r}\right)-\mathrm{x}_{\mathrm{i}} \arctan \frac{\mathrm{v}}{\mathrm{x}_{\mathrm{i}}^{2} \mathrm{r}}\right]_{\mathrm{x}_{\mathrm{b}}=-\mathrm{D}_{\mathrm{b}}-\mathrm{x}_{\mathrm{b}}}^{\mathrm{D}_{\mathrm{b}}-\mathrm{x}_{\mathrm{b}}}
\end{aligned}
$$

for distinct $\mathrm{i}, \mathrm{j}, \mathrm{k}$, where $\mathrm{e}_{\mathrm{i}}$ are the unit vectors for the $\mathrm{x}$, $\mathrm{y}, \mathrm{z}$ coordinate system.

If we look at the changing direction of the field as we move across a face, then we observe that the field vector only points towards the center of the cube at the center of each face, at the corners and at the center of each edge, which could also be deduced by symmetry arguments, refer Fig. 2.

\subsection{Orbits Around the Cube}

Could a moon or satellite form a stable orbit around such a cubic planet? We notice that there is a slightly greater gravitational force of attraction over the corners of the cube and hence an orbiting satellite will suffer significant perturbation in these areas, refer Fig. 3. A cube has three symmetry planes parallel to its faces, six symmetry planes in the diagonal plane and four asymmetry planes that contain the regular hexagonal cross section. It has been shown (Liu et al., 2011a), that periodic orbits can form in all three cases, although only planar-type orbits form when orbiting around the axis of symmetry, that is parallel to the faces or diagonally across the corners.

If we take the planar satellite orbits, around the faces and the centre of the edges, we produce the orbital Equation 8:

$$
\ddot{\mathrm{x}}=\mathrm{g}_{\mathrm{x}}, \ddot{\mathrm{y}}=\mathrm{g}_{\mathrm{y}}
$$

Solving this equation numerically for the specific case, of a satellite orbiting a cube with a side length equal to the diameter of the Earth, with an initial height of three Earth radii moving in the positive $\mathrm{x}$ direction with a velocity of $3.63 \mathrm{~km} \mathrm{~s}^{-1}$ gives the orbit shown in Fig. 4.

We find an orbital period of approximately $4.8 \mathrm{~h}$ and due to the interaction over the corners, the orbit is distorted from a perfect ellipse, creating a fairly rapid counterclockwise precession of the successive apogees as may also be seen in Fig. 4. The orbit does not close after the first orbit, but provided the precession amount is a rational fraction of the orbit, the orbit will eventually close. 


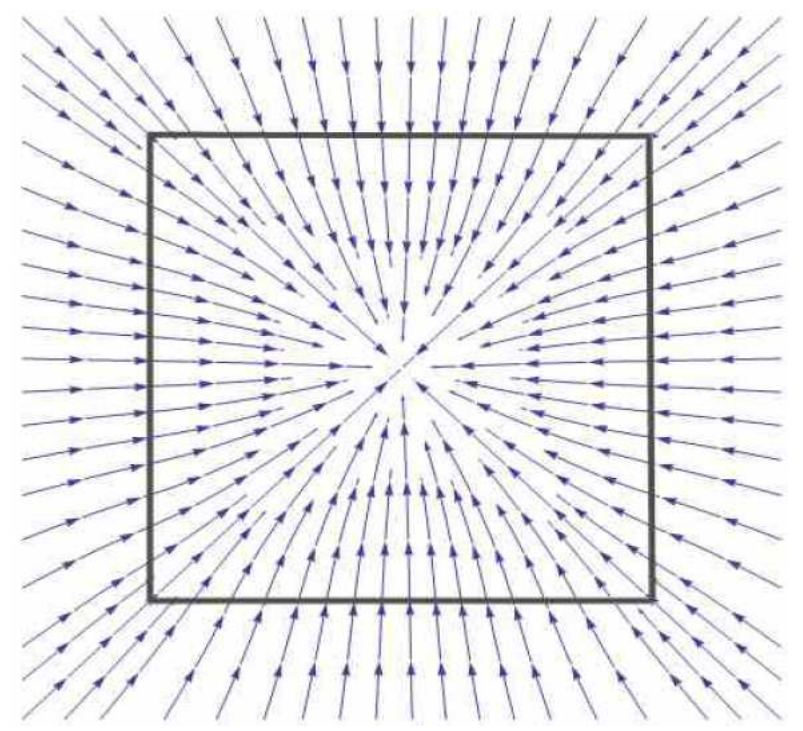

Fig. 2. The field through a cube sliced in half through the faces. We can observe the slight distortion of the field lines between the edges and the center of each face
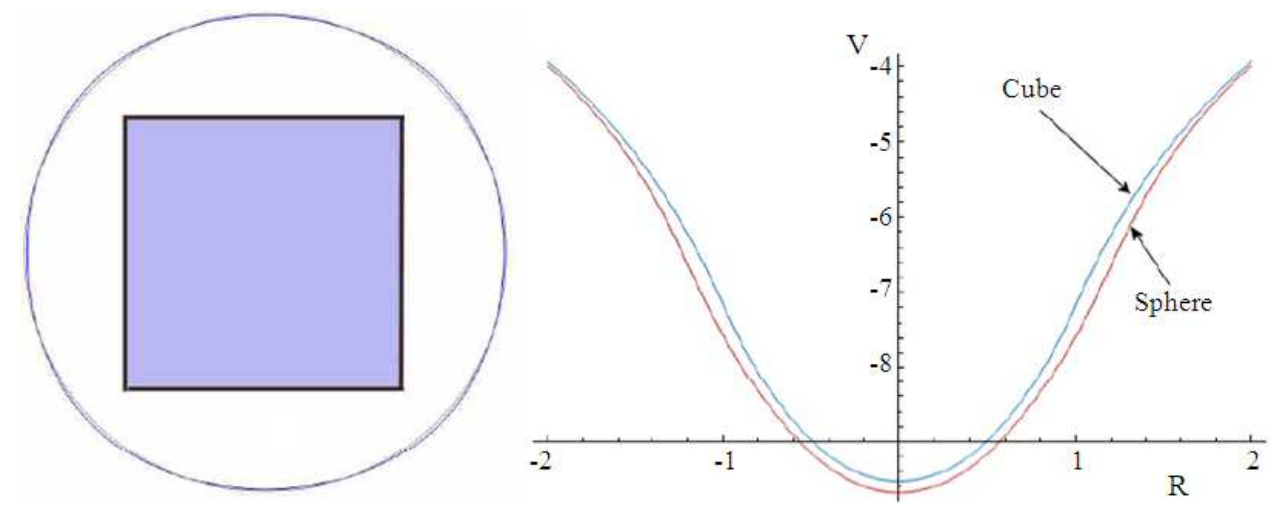

Fig. 3. The equipotential around the equator of the cube. Notice how the field is slightly stronger over the corners, indicated by the equipotential being shifted outwards compared to a perfect circle. When comparing the gravitational potential of a cube to a sphere of the same mass, because the sphere is a more compact object we find a deeper potential well, though the two potentials converge at larger distances as expected

Bertrand's theorem from classical mechanics states that 'The only central forces that result in closed orbits for all bound particles are the inverse-square law and Hooke's law.' (Goldstein et al., 2002) Due to the presence of a cuboid mass distribution the force field felt by the satellite is not inverse square and so we would not expect closed orbits. Alternatively, the potential of a cubic distribution of matter inside a cube can be expanded in a series of multipoles, that is, in a series $\mathrm{r}^{-\mathrm{k}}$, where $\mathrm{k}=1,2,3 \ldots$, which implies a precession of the perihelion.

By simulating a sequence of orbits of a satellite forwards in time, the Poincare section can be obtained by plotting $(\mathrm{x}, \dot{\mathrm{x}})$ as the particle crosses the $\mathrm{x}$-axis. This procedure may be repeated for different initial condition of the satellite though with the same value for the satellite energy, as shown in Fig. 5. Hence this method is useful when seeking to determine a stable orbit trajectory for a satellite of a given energy.

If we now investigate a satellite orbiting a rotating cube (Liu et al., 2011b) in the same direction as the cube rotation we find quite irregular orbits due to the significant perturbation over the corners of the cube when the satellite is near perigee that deflects the satellites orbit at seemingly irregular intervals (due to the differential rotation periods). 


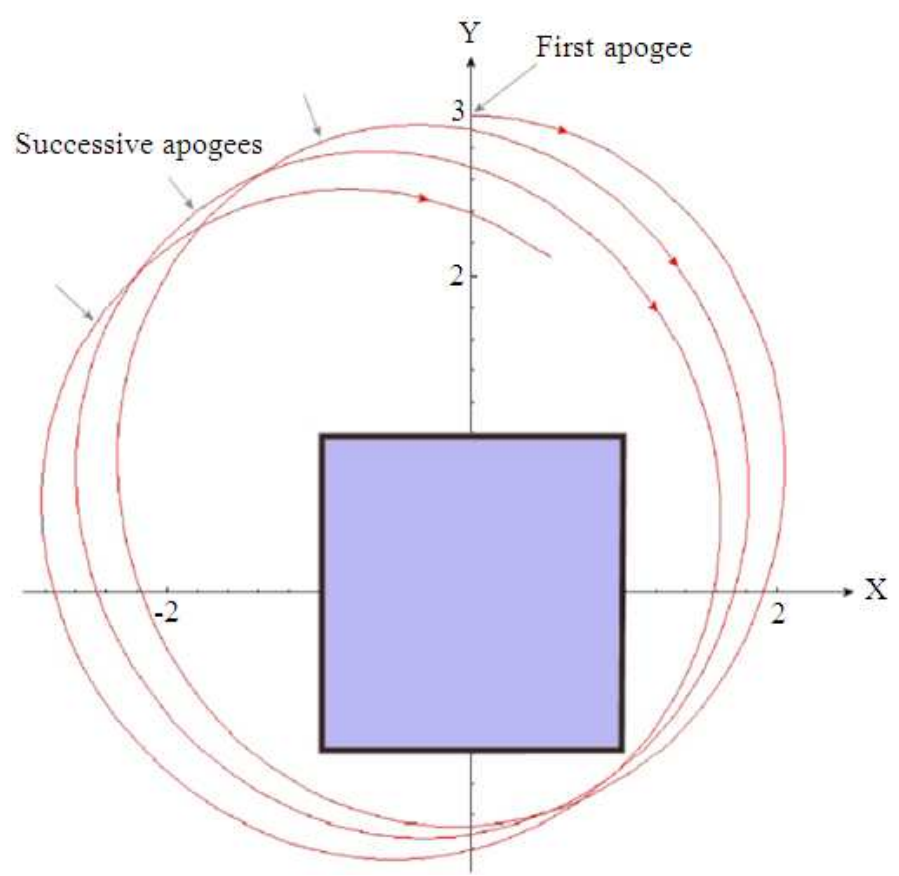

Fig. 4. A satellite orbiting around the equator of a static cube with a side length equal to the diameter of the Earth. Beginning with an satellite orbital radius of three Earth radii and a velocity of $3.63 \mathrm{~km} \mathrm{~s}^{-1}$, we find a period of approximately $4.8 \mathrm{~h}$, but with an orbit that precesses fairly rapidly, as shown by the counterclockwise movement of successive apogees

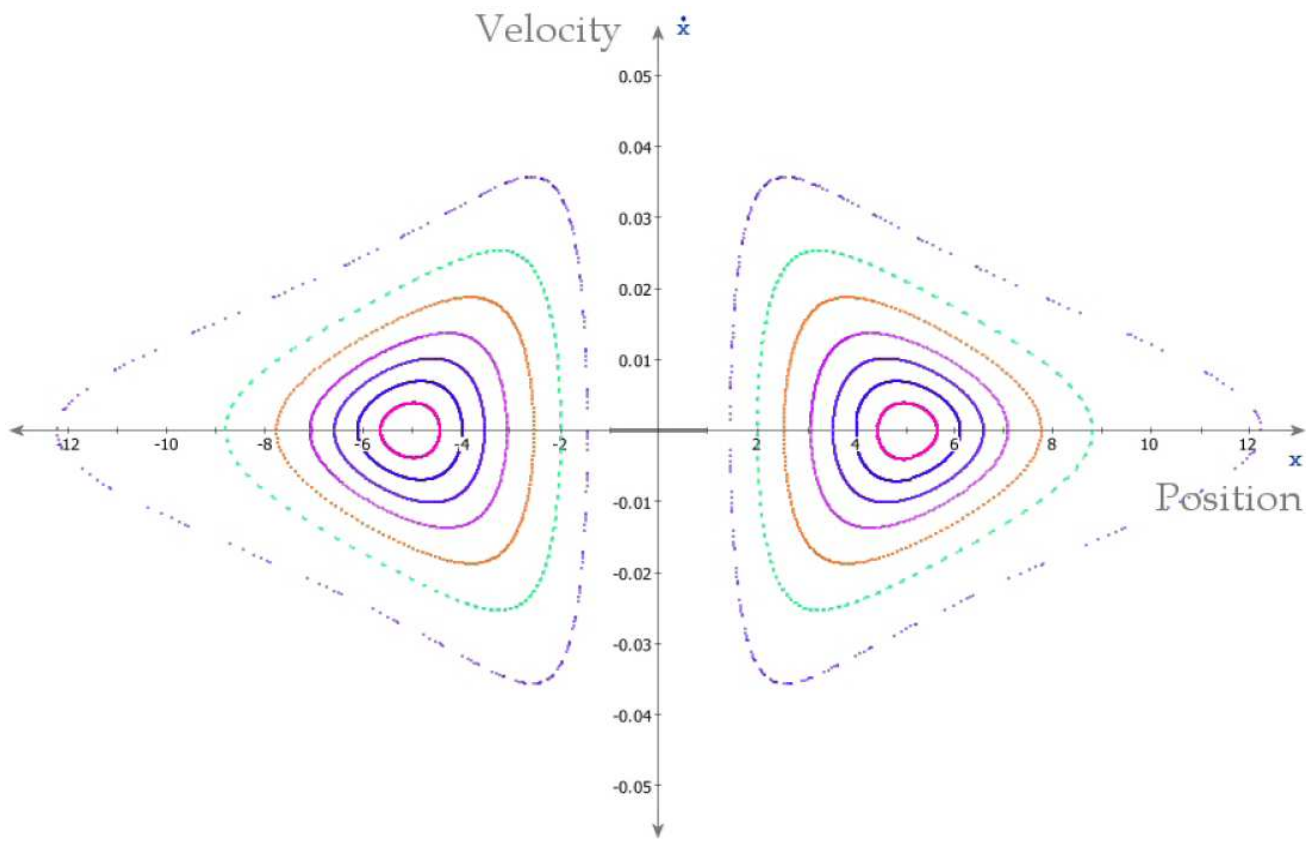

Fig. 5. The Poincare section, plotting the $x$ coordinate (when $y=0$ ) against its velocity in the $x$ direction, for satellites around the unit cube showing a closed orbit for this particular satellite energy at approximately five units 

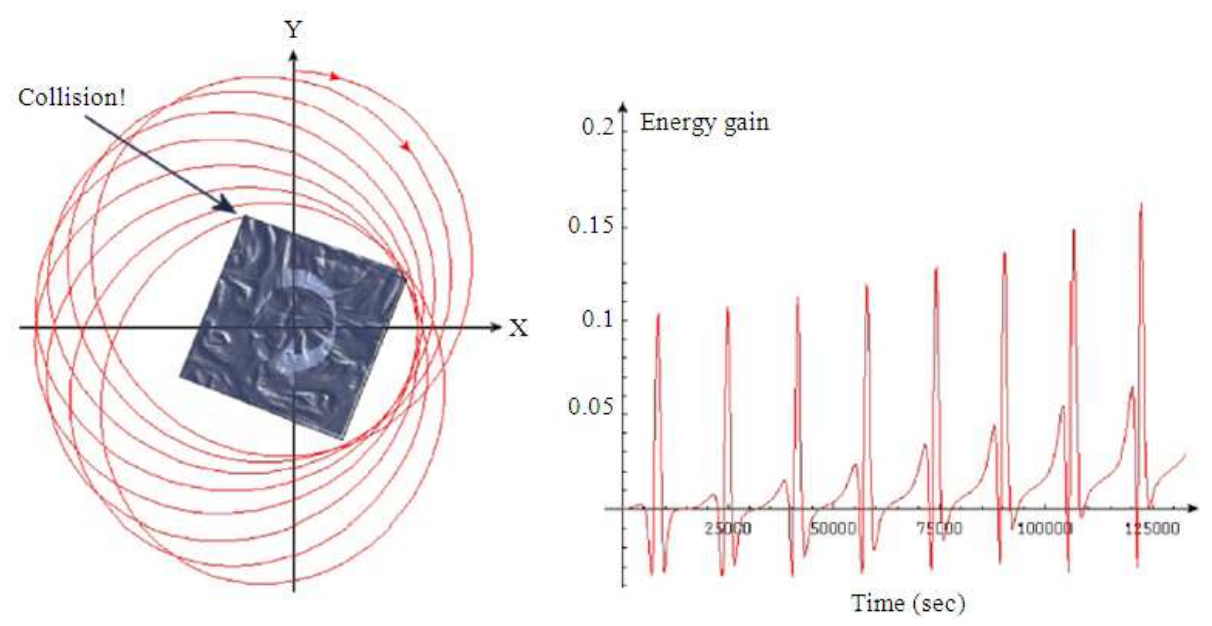

Fig. 6. Orbits around a cube rotating with a $10 \mathrm{~h}$. day, with a $4.8 \mathrm{~h}$. satellite orbit, close to a $2: 1$ resonance. With this configuration we find that the satellite rapidly acquires energy, colliding with the cube near the end of the eighth orbit as shown. From the graph of energy gain, we can see that by the $8^{\text {th }}$ orbit the satellite has a nearly $17 \%$ energy gain at perigee

That is, the stable orbits apparent on the Poincare section in Fig. 5, will now be deflected rhythmically from these positions, making orbits less stable. For example, with a cube rotation forming a $10 \mathrm{~h}$ day, so that the period is approximately double the satellite period of $4.8 \mathrm{~h}$, in order to highlight any resonance effects, then we find in the 8th orbit that the satellite now collides with the face of the cube, as shown in Fig. 6.

The satellite picks up energy through the well known slingshot effect, as can be seen in Fig. 6. This energy is being extracted from the cube, however as the satellite periodically goes out of resonance with the cube, the energy will be returned to the cube, so that the energy of the combined system is constant. It is not easily visible on Fig. 6 but the apogee of the satellite is in fact increasing by a few percent that reflects the energy gain.

As others have already discussed, a second planar configuration is possible with orbits diagonally over the corners of the cube, but if we allow more general orbits not aligned along these axes of symmetry then the orbits take on a more complex three-dimensional nature (Liu et al., 2011b).

\section{CONCLUSION}

We review in this study the nature of the gravitational field and satellite orbits that would form around a hypothetical cubic Earth-sized planet.

Calculating the gravitational potential and vector field for a cubic mass, we firstly find the shape of lakes that would form on the surface of such a cubic mass, shown in Fig 1. We then examine the possibility of stable orbits around such an object. Solving orbital equations numerically we find that the orbits are not closed in general but precess fairly rapidly due to interactions of the satellites with the mass in the corners of the cube. However by plotting the Poincare section for the satellite, as shown in Fig. 5, we can identify the initial conditions which will form a single closed orbit for a given satellite energy. Orbits are then calculated for the case where the cube is allowed to rotate, which produced resonance effects between the cube rotation and the satellite orbital period, causing previously stable satellite orbits to now crash into the cube, as shown in Fig. 6.

As an extension to our analysis involving satellite orbits under the assumption of Newtonian gravity, we could also calculate the general relativistic corrections due to the use of Einstein's theory of gravity. We expect, though, that these effects will only become significant for special situations, such as those involving high orbital speeds or a high rotation rate for the central body producing the Lense-Thirring effect, for example (Iorio et al., 2011; Iorio, 2005; Misner et al., 1973). As a further extension we might also consider the calculation of the energy levels determined from the Schrodinger equation, utilizing the classical cube potential $\mathrm{V}$, given by $\left(\frac{-\mathrm{h}}{2 \mathrm{~m}} \nabla^{2}+\mathrm{V}\right)|\psi\rangle=\mathrm{E}|\psi\rangle$.

Consider now a hypothetical cube $12,000 \times 12,000 \times 12,000 \mathrm{~km}^{3}$, approximately the size of 
the Earth, with the same volume of water and atmosphere as found on the Earth, then we would approximately half fill each face with water and have an atmosphere approximately $100 \mathrm{~km}$ thick similar to what is assumed for the atmosphere on the Earth before reaching space. In this case then the corners and the edges of the cube, would be like vast mountain ranges several thousand $\mathrm{km}$ high, with their tips extending out into free space. It would therefore be very difficult to cross these mountain ranges and hence we would have six nearly independent habitable zones on each face. There would presumably be permanent snow on the sides of these vast mountain ranges and people would live around the edges of the oceans on each face in a fairly narrow habitable zone only about $100 \mathrm{~km}$ wide as the cube faces rise rapidly through the atmosphere. Unfortunately climbing the approximately $3000 \mathrm{~km}$ high corners does not result in an improved view because the surface is still fiat in any observed direction. However the corners, being in free space, would be very suitable for launching satellites. One would also have approximately $\sqrt{2} \times 6000 \mathrm{~km}$ of downhill ski run from each corner, down to the centre of each face. In order to have a day night cycle we would also need the cube to be rotating. The sun would rise almost instantaneously over the face of a cube however, so that each face would need to be a single time zone and thus the cube as a whole would require four separate time zones, assuming the planet was rotating about the centre of an upper and lower face. The north and south faces in this case would be permanently frozen as they would receive no sunlight except that striking the oceans extending away from the surface of the cube, so there might be a permanent pool of liquid water at the two poles. Launching low orbit satellites around this cube needs special care in order to avoid certain orbital resonances that would create significant variations in the orbit (Berezovsky, 2011).

\section{REFERENCES}

Alberti, A. and C. Vidal, 2007. Dynamics of a particle in a gravitational field of a homogeneous annulus disk. Celestial Mechan. Dynamical Astron., 98: 75-93. DOI: $10.1007 / \mathrm{s} 10569-007-9071-\mathrm{z}$

Azevedo, C. and P. Ontaneda, 2007. On the existence of periodic orbits for the fixed homogeneous circle problem. J. Differ. Eq., 235: 341-365. DOI: 10.1016/j.jde.2006.12.012

Azevedo, C., H. Cabral and P. Ontaneda, 2005. On the fixed homogeneous circle problem. Cornell University Library.
Berezovsky, J., 2011. If the world were a cube, how would gravity be different? Case Western Reserve University.

Blesa, F., 2005. Periodic orbits around simple shaped bodies. Proceedings of the 9th International Conference Zaragoza-Pau on Applied Mathematics and Statistics, Sep. 19-21, Jaca, Spain, pp: 67-74.

Celnikier, L., 1990. Tidal braking of the s rotation-a study in cubism. Eur. J. Phys., 11: 60-60. DOI: 10.1088/0143-0807/11/1/011

Coggon, J.H., 1976. Magnetic and gravity anomalies of polyhedra. Geoexploration, 14: 93-105. DOI: 10.1016/0016-7142(76)90003-X

Fukushima, T., 2010. Precise computation of acceleration due to uniform ring or disk. Celestial Mechan. Dynamical Astron., 108: 339-356. DOI: 10.1007/s10569-010-9304-4

Garcia-Abdeslem, J., 2005. The gravitational attraction of a right rectangular prism with density varying with depth following a cubic polynomial. Geophysics, 70: J39-J42. DOI: 10.1190/1.2122413

Goldstein, H., C.P. Poole and J.L. Safko, 2002. Classical Mechanics. 3rd Edn., Addison-Wesley, United States, ISBN-10: 0201657023, pp: 638.

Gutierrez-Romero, S., J.F. Palacian and P. Yanguas, 2004. The invariant manifolds of a finite straight segment. Monogr. Real Acad. Cienc. Zaragoza, 25: 137-148.

Hansen, R.O., 1999. An analytical expression for the gravity field of a polyhedral body with linearly varying density. Geophysics, 64: 75-77. DOI: 10.1190/1.1444532

Iorio, L, H.I.M. Lichtenegger, M.L. Ruggiero and C. Corda, 2011. Phenomenology of the lense-thirring effect in the solar system. Astrophys. Space Sci., 331: 351-395. DOI: 10.1007/s10509-010-0489-5

Iorio, L., 2005. Is it possible to measure the lensethirring effect on the orbits of the planets in the gravitational field of the Sun? Astron. Astrophys., 431: 385-389. DOI: 10.1051/0004-6361:20041646

Iorio, L., 2007. Dynamical determination of the mass of the kuiper belt from motions of the inner planets of the Solar system. Mon. Not. Royal Astron. Soc., 375: 1311-1314.

Iorio, L., 2012. Orbital perturbations due to massive rings. Earth, Moon Planets, 108: 189-217. DOI: 10.1007/s11038-012-9391-1

Liu, X., H. Baoyin and X. Ma, 2011a. Periodic orbits in the gravity field of a fixed homogeneous cube. Astrophys. Space Sci., 334: 357-364. DOI: 10.1007/s10509-011-0732-8 
Liu, X., H. Baoyin and X. Ma, 2011b. Equilibria, periodic orbits around equilibria and heteroclinic connections in the gravity field of a rotating homogeneous cube. Astrophys. Space Sci., 333: 409-418. DOI: 10.1007/s10509-011-0669-y

MacMillan, W.D., 1958. Theoretical Mechanics: The Theory of the Potential. 1st Edn., Dover Publ., New York, pp: 469.

Michalodimitrakis, M. and G. Bozis, 1985. Bounded motion in a generalized two-body problem. Astrophys. Space Sci., 117: 217-225. DOI: 10.1007/BF00650148

Misner, C.W., K.S. Thorne and J.A. Wheeler, 1973. Gravitation. 1st Edn., Freeman, New York, ISBN-10:0716703440, pp: 1279.

Mufti, I.R., 2006a. Rapid determination of cube's gravity field. Geophys. Prospect., 21: 724-735. DOI: 10.1111/j.1365-2478.1973.tb00054.x

Mufti, I.R., 2006b. Iterative gravity modeling by using cubical blocks. Geophys. Prospect., 23: 163-198. DOI: $10.1111 / \mathrm{j} .1365-2478.1975 . t b 00688 . x$

Nagy, D., 1966. The gravitational attraction of a right rectangular prism. Geophysics, 31: 362-371. DOI: 10.1190/1.1439779

Najid, N.E. and E.H. Elourabi, 2012. Equilibria and stability around a straight rotating segment with a parabolic profile of mass density. Open Astron. J., 5: 19-25. DOI: $10.2174 / 1874381101205010019$

Najid, N.E., M. Zegoumou and E.H. Elourabi, 2012. Dynamical behavior in the vicinity of a circular anisotropic ring. Open Astron. J., 5: 54-60. DOI: 10.2174/1874381101205010054

Palacian, J.P., P. Yanguas and S. Gutierrez-Romero, 2006. Approximating the invariant sets of a finite straight segment near its collinear equilibria. SIAM J. Applied Dyn. Syst., 5: 12-29. DOI: 10.1137/040614517

Paul, M.K., 1974. The gravity effect of a homogeneous polyhedron for three-dimensional interpretation. Pure Applied Geophys., 112: 553-561. DOI: 10.1007/BF00877292
Riaguas, A., A. Elipe and M. Lara, 1999. Periodic orbits around a massive straight segment. Celestial Mechan. Dyn. Astron., 73: 169-178. DOI: 10.1023/A:1008399030624

Riaguas, A., A. Elipe and T. Lopez-Moratalla, 2001. Non-linear stability of the equilibria in the gravity field of a finite straight segment. Celestial Mechan. Dyn. Astron., 81: 235-248. DOI: 10.1023/A:1013217913585

Scheeres, D.J., B.G. Williams and J.K. Miller, 2000. Evaluation of the dynamic environment of an asteroid: Applications to 433 eros. J. Guidance, Control Dynam., 23: 466-475. DOI: 10.2514/2.4552

Scheeres, D.J., S.J. Ostro, R.S. Hudson and R.A. Werner, 1996. Orbits close to asteroid 4769 castalia. Icarus, 121: 67-87. DOI: 10.1006/icar.1996.0072

Scheeres, D.J., S.J. Ostro, R.S. Hudson, E.M. DeJong and S. Suzuki, 1998. Dynamics of orbits close to asteroid 4179 toutatis. Icarus, 132: 53-79. DOI: 10.1006/icar.1997.5870

Vankirk, W., 1885. The cubical planet: The surprising theory based on its alleged discovery.

Werner, R.A. and D.J. Scheeres, 1996. Exterior gravitation of a polyhedron derived and compared with harmonic and mascon gravitation representations of asteroid 4769 castalia. Celestial Mechan. Dyn. Astron., 65: 313-344. DOI: 10.1007/BF00053511

Werner, R.A., 1994. The gravitational potential of a homogeneous polyhedron or don't cut corners. Celestial Mechan. Dyn. Astron., 59: 253-278. DOI: 10.1007/BF00692875

Yu, Y. and H. Baoyin, 2012a. Generating families of 3D periodic orbits about asteroids. Mon. Not. Royal Astron. Soc., 427.1: 872-881. DOI: 10.1111/j.13652966.2012.21963.x

Yu, Y. and H. Baoyin, 2012b. Orbital dynamics in the vicinity of asteroid 216 kleopatra. Astron J., 143: 62-62. DOI: 10.1088/0004-6256/143/3/62

$\mathrm{Yu}, \mathrm{Y}$. and H. Baoyin, 2012c. Resonant orbits in the vicinity of asteroid 216 Kleopatra. Astrophys. Space Sci., 343: 75-82. DOI: 10.1007/s10509-012-1220-5 